\title{
G501C polymorphism of the oxidized LDL receptor gene is associated with albuminuria in Chinese essential hypertension patients
}

\author{
X.W. Hou ${ }^{1 *}$, Z.H. Hu ${ }^{2 *}$, Y. Cui ${ }^{3 *}$, W. Gao ${ }^{1}$, S.P. Sun ${ }^{1}$ and N.F. Wang ${ }^{1}$ \\ ${ }^{1}$ Department of Cardiology, \\ Hangzhou First Municipal Hospital \& Hangzhou Hospital, \\ Nanjing Medical University, Hangzhou, China \\ ${ }^{2}$ Department of Cardiovascular Disease, The Affiliated Tongji Hospital, \\ Tongji University, Shanghai, China \\ ${ }^{3}$ Department of Radiology, The Second Affiliated Hospital, \\ Shandong University, Jinan, China \\ *These authors contributed equally to this study. \\ Corresponding author: N.F. Wang \\ E-mail: beckhxw007@yahoo.com.cn
}

Genet. Mol. Res. 10 (4): 2710-2717 (2011)

Received March 28, 2011

Accepted September 1, 2011

Published October 3, 2011

DOI http://dx.doi.org/10.4238/2011.October.3.2

\begin{abstract}
Albuminuria is an independent predictor of renal and cardiovascular complications in hypertensive subjects. We previously showed that lectin-like oxidized low-density lipoprotein receptor 1 (OLR-1) polymorphisms at G501C are associated with susceptibility to essential hypertension and serum C-reactive protein levels. We have now investigated a possible association between OLR-1 polymorphisms at G501C, genotyped by PCR-RFLP, and severity of albuminuria in 307 hypertensive Chinese subjects and 225 age- and gender-matched controls. Urine albumin concentration/urine creatinine concentrations (ACR) were measured to evaluate the severity of albuminuria. Hypertensive subjects had a significantly higher frequency of the CC genotype and the $\mathrm{C}$ allele of the OLR-1 polymorphism than controls; this was also true for hypertensive subjects with macroalbuminuria and microalbuminuria
\end{abstract}


compared to those with normoalbuminuria. The mean ACR levels and mean serum C-reactive protein levels in CC carriers were significantly higher than in GG and GC carriers. There was a significant, positive correlation between serum hs-C-reactive protein levels and ACR levels. We conclude that OLR-1 polymorphisms at G501C affect the severity of albuminuria in essential hypertension patients.

Key words: Albuminuria; Polymorphisms; Essential hypertension; Lectin-like oxidized low-density lipoprotein receptor 1

\section{INTRODUCTION}

The kidney is one of the target organs of arterial hypertension. Renal impairment was present in more than $30 \%$ of patients with essential hypertension. Increased urinary albumin excretion is a marker for renal impairment. Prospective and epidemiologic studies have documented that albuminuria is a predictive factor for all-cause and cardiovascular mortality in subjects with hypertension as well as in the general population, independently of other traditional risk factors (Yuyun et al., 2004; Klausen et al., 2009). In patients with hypertension, albuminuria is suggested to be an independent predictor of renal and cardiovascular complications and these subjects need an aggressive approach of cardiovascular risk management (Viazzi et al., 2010). A prospective long-term study (MARPLE Study) showed that microalbuminuria and tubular proteinuria are risk predictors of cardiovascular morbidity and mortality in essential hypertension (Schrader et al., 2006).

Growing evidence suggests that endothelial dysfunction plays a key role in the renal impairment in subjects with hypertension (Klausen et al., 2007; Cerasola et al., 2008; Perticone et al., 2010). The Telmisartan versus Ramipril in renal ENdothelium DYsfunction (TRENDY) trial suggested endothelial function of the renal vasculature, as assessed by basal nitric oxide activity, as a therapeutic target in patients with hypertension and type 2 diabetes (Basi and Lewis, 2006). Albuminuria is suggested to be a marker of local (renal) endothelial dysfunction and may contribute to renal impairment independently of other risk factors (Perticone et al., 2007).

The lectin-like oxidized low-density lipoprotein (ox-LDL) receptor 1, encoded by the OLR-1 gene, is the major scavenger receptor for ox-LDL abundantly expressed in endothelial cells, which has recently been determined to play a notable role in the initiation and progression of endothelial dysfunction (Cominacini et al., 2003; Cilingiroglu and Ozer, 2005; Ogura et al., 2009).

The association of OLR-1 polymorphisms with several cardiovascular conditions including atherosclerosis, acute myocardial infarction, coronary artery disease, and ischemic stroke has been reported (Tatsuguchi et al., 2003; Ohmori et al., 2004; Mango et al., 2005; Hattori et al., 2006; Kurnaz et al., 2009). Tatsuguchi et al. (2003) identified a single nucleotide polymorphism (SNP) in the OLR-1 gene, G-to-C at position 501 in the open reading frame, which results in a mis-sense mutation of Lys to Asn at position 167 (K167N). Our previous study showed that the OLR-1 polymorphisms at G501C were associated with susceptibility to essential hypertension and with the serum C-reactive protein (CRP) level in a Chinese population (Hou et al., 2008). However, the role of OLR-1 polymorphisms in the renal impairment in essential hypertensive subjects has not been previously studied. In this study, we investigated the association between the OLR-1 polymorphisms at G501C and the severity of albuminuria in hypertensive subjects. 


\section{SUBJECTS AND METHODS}

\section{Subjects}

From January 2006 to January 2009, 307 consecutive patients with essential hypertension and 225 age- and gender-matched controls were enrolled. Hypertension was defined according to fifth Joint Committee on Detection, Evaluation and Treatment of High Blood Pressure criteria. None of the hypertensive subjects had received any antihypertensive therapy at enrollment. Patients with secondary hypertension, neoplastic, hepatic, and/or renal disease, chronic heart failure (NYHA classes III and IV), diabetes mellitus, renal failure, severe obesity, disabling diseases such as dementia were excluded from the study. The Ethics Committee of our institution approved this study and written informed consent was obtained from each participant.

\section{Albuminuria}

A previous study reported that the albumin-to-creatinine ratio (ACR) is a good measure of albuminuria (Gerber et al., 2006). We adapted ACR in this study to evaluate renal impairment. The ACR was calculated as urine albumin concentration $(\mathrm{mg} / \mathrm{L}) /$ urine creatinine concentration $(\mathrm{mM})$. Creatinine levels were determined by the routine Jaffe reaction and albumin concentration by a commercially available radioimmunoassay kit. Albuminuria was categorized as normoalbuminuria (ACR $<30 \mathrm{mg} / \mathrm{g}$ ), microalbuminuria (ACR $=30-299 \mathrm{mg} / \mathrm{g}$ ), or macroalbuminuria (ACR $\geq 300 \mathrm{mg} / \mathrm{g}$ ).

\section{Clinical characteristics}

Body mass index (BMI) was calculated using the formula: $\mathrm{BMI}=$ weight $(\mathrm{kg}) / \mathrm{height}$ $\left(\mathrm{m}^{2}\right)$. Blood urea nitrogen, electrolytes, uric acid, triglycerides, total and high-density lipoprotein cholesterol, and other standard chemistry evaluations were performed on serum according to routine methods. LDL cholesterol was calculated using Friedewald's formula, and creatinine clearance was calculated using Cockroft's formula. High-sensitivity CRP (hs-CRP) was detected according to the kit protocol.

\section{Statistical analysis}

The statistical analysis was performed using the SPSS Statistical Package, version 16.0. The mean values of all data were used for analysis and are shown as means $\pm \mathrm{SD}$. The demographic and clinical characteristic comparisons between hypertensive subjects and controls were performed using the Student test and the chi-square test. The genotype distributions and allele frequencies in hypertensive subjects and controls as well as among hypertensives with different ACR levels were performed with the chi-square test, as appropriate. The correlation between CRP and ACR was performed with Spearman analyses.

\section{RESULTS}

Among all hypertensive subjects, there were 183 subjects with normoalbuminuria, 96 
with microalbuminuria and 28 with macroalbuminuria. Table 1 summarizes the demographic and clinical characteristics of all hypertensive cases and controls. No significant differences were observed with respect to age, gender, smoker number, serum triglyceride, and total cholesterol between hypertensive subjects and controls. Meanwhile, the hypertensive group had a significantly higher blood pressure, BMI and serum hs-CRP concentration compared to the control group.

Table 1. Clinical characteristics of hypertensive and control subjects.

\begin{tabular}{lccc}
\hline & Hypertensives & Controls & P \\
\hline Men (\%) & 55.7 & 54.3 & NS \\
Age (years) & $49.7 \pm 8.2$ & $49.3 \pm 6.6$ & NS \\
BMI (kg/m $\left.{ }^{2}\right)$ & $25.3 \pm 2.6$ & $14.1 \pm 1.7$ & 0.02 \\
SBP (mmHg) & $145.6 \pm 11.4$ & $117.6 \pm 9.3$ & $<0.001$ \\
DBP (mmHg) & $97.5 \pm 7.8$ & $83.8 \pm 9.1$ & $<0.001$ \\
Smoker $(\%)$ & 46.3 & 45.9 & NS \\
Cholesterol (mM) & $6.12 \pm 2.14$ & $6.19 \pm 1.31$ & NS \\
LDL (mM) & $2.25 \pm 0.44$ & $2.18 \pm 0.51$ & 0.052 \\
Triglyceride (mM) & $1.67 \pm 0.49$ & $1.65 \pm 0.84$ & NS \\
hs-CRP (mg/L) & $2.18 \pm 0.25$ & $1.34 \pm 0.26$ & 0.002 \\
\hline BMI = body mass index; SBP, DBP $=$ systolic and diastolic blood pressure; LDL $=10$ low-density lipoprotein; hs-CRP \\
$=$ high-sensitivity C-reactive protein; NS = non-significant.
\end{tabular}

Table 2 summarizes the genotype distributions and allele frequencies in hypertensive subjects and controls. Both genotype distributions and allele frequencies in patients and controls were compatible with Hardy-Weinberg's equilibrium. Similar to our previous published data, the genotype distributions and allele frequencies of OLR-1 polymorphism were significantly different between Chinese hypertensive subjects and controls. Hypertensive cases had a significantly higher prevalence of the $\mathrm{CC}$ genotype than controls $(24.4 v s 8.0 \%, \mathrm{P}<0.001)$. The $\mathrm{C}$ allele frequency in hypertensive subjects was higher than in controls $(41.0 \mathrm{vs} 18.2 \%, \mathrm{P}<0.001)$.

\begin{tabular}{|c|c|c|c|c|c|}
\hline & Hypertensives $(\mathrm{N}=307)$ & Controls $(\mathrm{N}=225)$ & Chi-square & d.f. & $\mathrm{P}$ \\
\hline \multicolumn{6}{|c|}{ Genotypes } \\
\hline $\mathrm{GG}$ & $130(42.3 \%)$ & $116(51.6 \%)$ & 24.2 & 2 & $<0.001$ \\
\hline $\mathrm{GC}$ & $102(33.2 \%)$ & $91(40.4 \%)$ & & & \\
\hline $\mathrm{CC}$ & $75(24.5 \%)$ & $18(8.0)$ & & & \\
\hline \multicolumn{6}{|c|}{ Alleles } \\
\hline G & $362(59.0 \%)$ & $323(71.8 \%)$ & 26.7 & 1 & $<0.001$ \\
\hline $\mathrm{C}$ & $252(41.0 \%)$ & $127(18.2 \%)$ & & & \\
\hline
\end{tabular}

d.f. $=$ degrees of freedom.

Table 3 shows the genotype distributions and the allele frequencies of OLR-1 polymorphism in all hypertensive subjects according to their ACR level. A significant difference was noted in the genotype distributions and allele frequencies of OLR-1 polymorphisms among hypertensive subjects with normoalbuminuria, microalbuminuria and macroalbuminuria. The prevalence of the CC type was significantly higher in subjects with macroalbuminuria and microalbuminuria than in those with normoalbuminuria ( $71.4 v s 14.8 \%$ and $29.2 v s$ $14.8 \%, \mathrm{P}<0.001$, respectively). Correspondingly, the $\mathrm{C}$ allele frequencies in subjects with macroalbuminuria and microalbuminuria were significantly higher than in those with normoalbuminuria (80.4 vs $33.6 \%$ and 43.8 vs $33.6 \%, \mathrm{P}<0.001)$. 


\begin{tabular}{|c|c|c|c|c|c|c|}
\hline & $\begin{array}{l}\text { Normoalbuminuria } \\
\quad(\mathrm{N}=183)\end{array}$ & $\begin{array}{l}\text { Microalbuminuria } \\
(\mathrm{N}=96)\end{array}$ & $\begin{array}{l}\text { Macroalbuminuria } \\
\quad(\mathrm{N}=28)\end{array}$ & Chi-square & d.f. & $P$ \\
\hline \multicolumn{7}{|c|}{ Genotypes } \\
\hline GG & $87(47.5 \%)$ & $40(41.7 \%)$ & $3(10.7 \%)$ & 44.7 & 4 & $<0.001$ \\
\hline GC & $69(37.7 \%)$ & $28(29.2 \%)$ & $5(17.9 \%)$ & & & \\
\hline $\mathrm{CC}$ & $27(14.8 \%)$ & $28(29.2 \%)$ & $20(71.4 \%)$ & & & \\
\hline \multicolumn{7}{|c|}{ Alleles } \\
\hline G & $243(66.4 \%)$ & $108(56.2 \%)$ & $11(19.6 \%)$ & 21.3 & 2 & $<0.001$ \\
\hline C & $123(33.6 \%)$ & $84(43.8 \%)$ & $45(80.4 \%)$ & & & \\
\hline
\end{tabular}

Figure 1 shows the mean ACR level in each genotype carriers among hypertensive subjects. The mean ACR level in CC carriers was significantly higher than in GC and GG carriers $(221.5 \pm 98.5$ vs $123.5 \pm 45.5$ and $112.6 \pm 34.8$, both $\mathrm{P}<0.05)$.

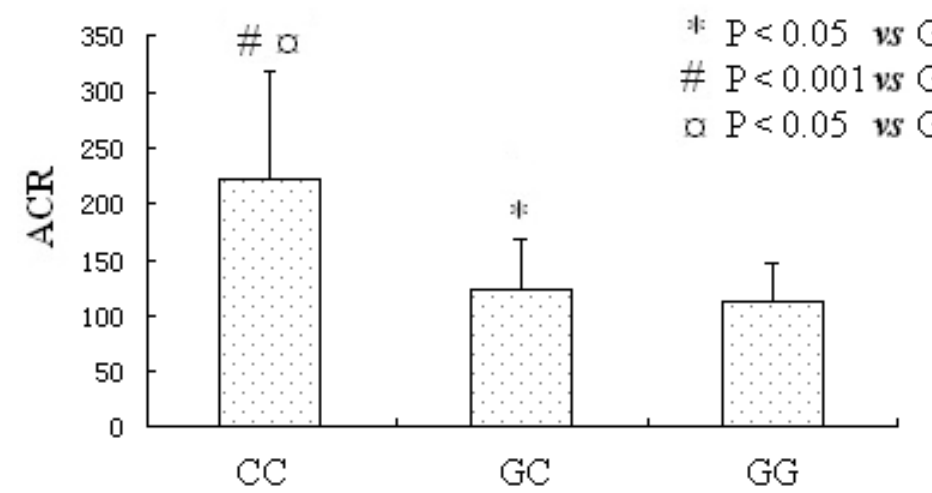

Figure 1. Mean albumin-to-creatinine ratio (ACR) level in hypertensive subjects according to their genotypes.

Figure 2 shows the mean hs-CRP level in each genotype carriers among hypertensive subjects. The mean hs-CRP level in CC carriers was significantly higher than in GG and GC carriers $(1.8 \pm 0.35$ vs $1.1 \pm 0.2$ and $1.2 \pm 0.3 \mathrm{mg} / \mathrm{L}$, both $\mathrm{P}<0.05)$.

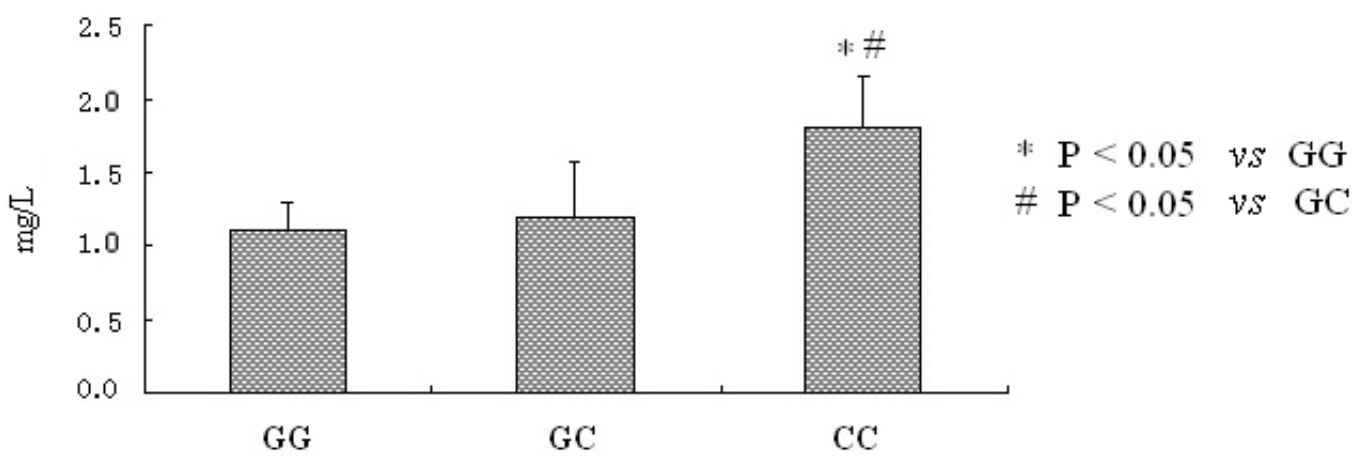

Figure 2. Mean high-sensitivity C-reactive protein (hs-CRP) levels in hypertensive subjects according to their genotypes. 
We next evaluated the correlation between the serum CRP levels and the ACR levels by Spearman analyses. Our results showed that there was a positive correlation between serum hs-CRP levels and ACR levels $(r=0.845, \mathrm{P}<0.001)$.

\section{DISCUSSION}

In the present study, we investigated the association between the G501C polymorphism of OLR-1 and albuminuria in Chinese subjects with essential hypertension. We found that the CC genotype represents a higher prevalence in subjects with macroalbuminuria and microalbuminuria than in those with normoalbuminuria, suggesting that this genotype is associated with severity of albuminuria in essential hypertension subjects.

Enhanced renal expression of OLR-1 is reported to be involved in the progression of chronic renal failure in rats (Ueno et al., 2003). Inhibition of OLR-1 mRNA expression in human umbilical vein endothelial cells decreased apoptosis induced by angiotensin II and oxLDL, thus improving endothelial cell function (Ueno et al., 2009). The OLR-1 up-regulation in the kidney of Dahl salt-sensitive rats was parallel to glomerulosclerotic changes and renal dysfunction, suggesting a possible pathogenetic role for renal OLR-1 in the progression to hypertensive glomerulosclerosis (Nagase et al., 2000). In subjects with diabetic mellitus, increased expression of OLR-1 mRNA in the tubulointerstitial area may be closely linked to the development and progression of diabetic nephropathy and tubulointerstitial damage (Yamamoto et al. 2009). The role of OLR-1 in the development of angiotensin II-induced hypertension and subsequent renal damage was recently reported (Hu et al., 2009).

Previous studies investigated the association between the OLR-1 G501C polymorphisms and cardiovascular disease, but with conflicting results. Trabetti et al. (2006) found that the G501C G/G genotype is associated with an increased number of obstructed coronary vessels while others found that the G501C SNP is inversely associated with the severity of CAD (Tatsuguchi et al., 2003; Ohmori et al., 2004). Our previous study (Hou et al., 2008) suggested that the CC genotype of the G501C genotype was an independent risk factor for essential hypertension $(\mathrm{OR}=3.036,95 \% \mathrm{CI}=1.572-6.174, \mathrm{P}=0.016)$; whereas, the $\mathrm{CC}$ homozygous carriers have significantly higher CRP concentration than GG and GC carriers. To date, little research has been carried out regarding the association between the OLR-1 gene variation and kidney impairment in subjects with essential hypertension. Our current data, in combination with our previous data, suggest the importance of G501C polymorphism of OLR-1 in predicting the susceptibility and renal impairment of essential hypertension in the Chinese population.

The ORL-1 polymorphisms at G501C influence the ox-LDL-induced biosynthesis of ORL-1. A recent biochemical analysis showed that this genetic variant of ORL-1 changed oxLDL binding and subsequent ERK signal pathway activation by as much as 30\% (Biocca et al., 2009). Electrostatic interactions between basic residues in this domain and negatively charged residues in ox-LDL are critical for OLR-1 activity, and substitution of these residues may cause reduced ox-LDL binding and internalization. As ORL-1 mediates ox-LDL-induced atherosclerosis and endothelial dysfunction, it is plausible that changed ORL-1 activity by G501C polymorphisms directly affects the endothelial dysfunction induced by ox-LDL in vivo.

The OLR-1 gene variation has been reported to regulate soluble OLR-1 levels in plasma (Brinkley et al., 2008). Brinkley et al. (2008) found that the G501C polymorphisms affect plasma OLR-1 expression. The GG genotype of ORL-1 was associated with lower soluble 
OLR-1 levels in their study (Brinkley et al., 2008). According to their findings, we postulate that the detrimental effect of the CC genotype might be a result of a higher ORL-1 plasma level. Inflammation is another early feature of human hypertension. Increased serum CRP level is considered to be a forerunner of hypertension in the general population. In patients with untreated essential hypertension, increased hs-CRP levels are associated with microalbuminuria, suggesting the involvement of inflammation in vascular and kidney damage (Tsioufis et al., 2010). In our study, we found that the CC genotype is associated with elevated CRP levels, reflecting a higher inflammation status in CC genotype carriers. Li and co-workers (2004) found that CRP stimulates OLR-1 protein expression in endothelial cells, thus enhancing the uptake of ox-LDL, suggesting that CRP may trigger and enhance the ox-LDL-induced vessel endothelial dysfunction via stimulating OLR-1 protein expression on vascular endothelium. At the same time, we observed a positive correlation between the ACR and CRP levels, strengthening the idea that albuminuria and CRP are of relevance in essential hypertension, particularly in individuals with albuminuria.

Several limitations need to be addressed in this study. First, this is a relatively smallscale study. Future studies of a larger scale are necessary to elucidate the role of ORL-1 gene variants in essential hypertension. Second, we did not detect the plasma ORL-1 level to directly manifest the effect of ORL-1 G501C polymorphisms on its downstream protein expression.

\section{REFERENCES}

Basi S and Lewis JB (2006). Microalbuminuria as a target to improve cardiovascular and renal outcomes. Am J. Kidney Dis. 47: 927-946.

Biocca S, Falconi M, Filesi I, Baldini F, et al. (2009). Functional analysis and molecular dynamics simulation of LOX-1 K167N polymorphism reveal alteration of receptor activity. PLoS One 4: e4648.

Brinkley TE, Kume N, Mitsuoka H, Brown MD, et al. (2008). Variation in the human lectin-like oxidized low-density lipoprotein receptor 1 (LOX-1) gene is associated with plasma soluble LOX-1 levels. Exp. Physiol. 93: 1085-1090.

Cerasola G, Mule G, Cottone S, Nardi E, et al. (2008). Hypertension, microalbuminuria and renal dysfunction: the Renal Dysfunction in Hypertension (REDHY) study. J. Nephrol. 21: 368-373.

Cilingiroglu M and Ozer K (2005). The lectin-like oxidized low-density lipoprotein receptor and its role in atherosclerosis. Curr. Atheroscler. Rep. 7: 103-107.

Cominacini L, Fratta PA, Garbin U, Pastorino A, et al. (2003). The platelet-endothelium interaction mediated by lectinlike oxidized low-density lipoprotein receptor-1 reduces the intracellular concentration of nitric oxide in endothelial cells. J. Am. Coll. Cardiol. 41: 499-507.

Gerber LM, Schwartz JE and Pickering TG (2006). Albumin-to-creatinine ratio predicts change in ambulatory blood pressure in normotensive persons: a 7.5-year prospective study. Am. J. Hypertens. 19: 220-226.

Hattori H, Sonoda A, Sato H, Ito D, et al. (2006). G501C polymorphism of oxidized LDL receptor gene (OLR1) and ischemic stroke. Brain Res. 1121: 246-249.

Hou XW, Wang LF, Wang N, Pang D, et al. (2008). The G501C polymorphism of oxidized LDL receptor gene [OLR-1] is associated with susceptibility and serum C-reactive protein concentration in Chinese essential hypertensives. Clin. Chim. Acta 388: 200-203.

Hu C, Kang BY, Megyesi J, Kaushal GP, et al. (2009). Deletion of LOX-1 attenuates renal injury following angiotensin II infusion. Kidney Int. 76: 521-527.

Klausen KP, Parving HH, Scharling H and Jensen JS (2007). The association between metabolic syndrome, microalbuminuria and impaired renal function in the general population: impact on cardiovascular disease and mortality. J. Intern. Med. 262: 470-478.

Klausen KP, Parving HH, Scharling H and Jensen JS (2009). Microalbuminuria and obesity: impact on cardiovascular disease and mortality. Clin. Endocrinol. 71: 40-45.

Kurnaz O, Aydogan HY, Isbir CS, Tekeli A, et al. (2009). Is LOX-1 K167N polymorphism protective for coronary artery disease? In Vivo 23: 969-973.

Li L, Roumeliotis N, Sawamura T and Renier G (2004). C-reactive protein enhances LOX-1 expression in human aortic 
endothelial cells: relevance of LOX-1 to C-reactive protein-induced endothelial dysfunction. Circ. Res. 95: 877-883.

Mango R, Biocca S, del VF, Clementi F, et al. (2005). In vivo and in vitro studies support that a new splicing isoform of OLR1 gene is protective against acute myocardial infarction. Circ. Res. 97: 152-158.

Nagase M, Kaname S, Nagase T, Wang G, et al. (2000). Expression of LOX-1, an oxidized low-density lipoprotein receptor, in experimental hypertensive glomerulosclerosis. J. Am. Soc. Nephrol. 11: 1826-1836.

Ogura S, Kakino A, Sato Y, Fujita Y, et al. (2009). Lox-1: the multifunctional receptor underlying cardiovascular dysfunction. Circ. J. 73: 1993-1999.

Ohmori R, Momiyama Y, Nagano M, Taniguchi H, et al. (2004). An oxidized low-density lipoprotein receptor gene variant is inversely associated with the severity of coronary artery disease. Clin. Cardiol. 27: 641-644.

Perticone F, Maio R, Tripepi G, Sciacqua A, et al. (2007). Microalbuminuria, endothelial dysfunction and inflammation in primary hypertension. J. Nephrol. 20 (Suppl 12): S56-S62.

Perticone F, Maio R, Perticone M, Sciacqua A, et al. (2010). Endothelial dysfunction and subsequent decline in glomerular filtration rate in hypertensive patients. Circulation 122: 379-384.

Schrader J, Luders S, Kulschewski A, Hammersen F, et al. (2006). Microalbuminuria and tubular proteinuria as risk predictors of cardiovascular morbidity and mortality in essential hypertension: final results of a prospective longterm study (MARPLE Study)*. J. Hypertens. 24: 541-548.

Tatsuguchi M, Furutani M, Hinagata J, Tanaka T, et al. (2003). Oxidized LDL receptor gene (OLR1) is associated with the risk of myocardial infarction. Biochem. Biophys. Res. Commun. 303: 247-250.

Trabetti E, Biscuola M, Cavallari U, Malerba G, et al. (2006). On the association of the oxidised LDL receptor 1 (OLR1) gene in patients with acute myocardial infarction or coronary artery disease. Eur. J. Hum. Genet. 14: 127-130.

Tsioufis C, Dimitriadis K, Andrikou E, Thomopoulos C, et al. (2010). ADMA, C-reactive protein, and albuminuria in untreated essential hypertension: a cross-sectional study. Am. J. Kidney Dis. 55: 1050-1059.

Ueno T, Kaname S, Takaichi K, Nagase M, et al. (2003). LOX-1, an oxidized low-density lipoprotein receptor, was upregulated in the kidneys of chronic renal failure rats. Hypertens Res. 26: 117-122.

Ueno T, Fukuda N, Tsunemi A, Yao EH, et al. (2009). A novel gene silencer, pyrrole-imidazole polyamide targeting human lectin-like oxidized low-density lipoprotein receptor-1 gene improves endothelial cell function. J. Hypertens. 27: 508-516.

Viazzi F, Leoncini G, Conti N, Tomolillo C, et al. (2010). Microalbuminuria is a predictor of chronic renal insufficiency in patients without diabetes and with hypertension: the MAGIC study. Clin. J. Am. Soc. Nephrol. 5: 1099-1106.

Yamamoto N, Toyoda M, Abe M, Kobayashi T, et al. (2009). Lectin-like oxidized LDL receptor-1 (LOX-1) expression in the tubulointerstitial area likely plays an important role in human diabetic nephropathy. Intern. Med. 48: 189-194.

Yuyun MF, Khaw KT, Luben R, Welch A, et al. (2004). Microalbuminuria independently predicts all-cause and cardiovascular mortality in a British population: The European Prospective Investigation into Cancer in Norfolk (EPIC-Norfolk) population study. Int. J. Epidemiol. 33: 189-198. 